\title{
L'émergence d'une scène politique : mouvement ouvrier et mouvements de réforme urbaine à Montréal au tournant du siècle - Essai d'interprétation
}

\section{Annick Germain}

Volume 37, numéro 2, septembre 1983

Travailleurs et mouvements sociaux

URI : https://id.erudit.org/iderudit/304153ar

DOI : https://doi.org/10.7202/304153ar

Aller au sommaire du numéro

Éditeur(s)

Institut d'histoire de l'Amérique française

ISSN

0035-2357 (imprimé)

1492-1383 (numérique)

Découvrir la revue

Citer cet article

Germain, A. (1983). L'émergence d'une scène politique : mouvement ouvrier et mouvements de réforme urbaine à Montréal au tournant du siècle - Essai d'interprétation. Revue d'histoire de l'Amérique française, 37(2), 185-199.

https://doi.org/10.7202/304153ar 


\section{L'ÉMERGENCE D'UNE SCÈNE POLITIQUE: MOUVEMENT OUVRIER ET MOUVEMENTS DE RÉFORME URBAINE À MONTRÉAL AU TOURNANT DU SIËCLE \\ — ESSAI D'INTERPRÉTATION -}

ANNICK GERMAIN

\section{Introduction}

L'analyse de la scène politique montréalaise au tournant du siècle (1880-1920) revêt une importance décisive dans la connaissance de l'histoire du mouvement ouvrier québécois. C'est en effet à Montréal que les forces ouvrières réalisent des percées politiques spectaculaires: fondation du Parti ouvrier de Montréal (1899), élection d'un député ouvrier au Parlement fédéral (1906), élection d'un chef ouvrier aux plus hautes instances du gouvernement municipal (1910), prolifération des clubs ouvriers municipaux dans les principaux quartiers de la ville, etc. La scène politique locale représente d'ailleurs, au début du siècle, un lieu stratégique crucial dans le traitement des problèmes sociaux: les problèmes de chômage, de pauvreté, de logement, de santé publique, ainsi que tout ce qu'on appelle à l'époque les services d'utilité publique (transport, chauffage, eau, éclairage) sont en effet négociés au niveau local. C'est dire que tout ce qui concerne les conditions de vie des citadins relève des instances locales publiques et privées ${ }^{1}$. Bien plus, les relations industrielles (ouvriers-patronat) concernent jusqu'à un certain point les autorités municipales. Les niveaux de gouvernement supérieurs réaffirmeront à plusieurs reprises les responsabilités qui incombent aux autorités municipales en cas de conflit ouvrier ${ }^{2}$. Bref, indépendamment de la capacité de gestion effective du gouvernement local, c'est dans la ville qu'affleurent les contradictions sociales majeures, c'est là que se cristallise l'aménagement des rapports sociaux. À Montréal, comme dans la plupart des grandes villes nord-américaines, de nouvelles forces sociales s'organisent sur la scène politique:

\footnotetext{
1 Le tournant du siècle représente une période charnière entre la tradition philanthropique et l'intervention de l'État dans la prise en charge des problèmes sociaux. Le pouvoir local ne se confond donc pas avec le pouvoir municipal.

2 Pendant la période étudiée, des personnalités montréalaises telles que R. Préfontaine, G. Stephens, M. Martin et J. Cochrane, seront amenées à assurer l'arbitrage de conflits ouvriers importants. Conscients, toutefois, du capital politique sous-tendant ces enjeux, les gouvernements provincial et fédéral délégueront discrètement quelques émissaires, à chaque occasion.
} 
mouvement ouvrier ${ }^{3}$ et mouvements de réforme urbaine animent la politique montréalaise au tournant du siècle.

Il est alors assez paradoxal de constater la pauvreté des débats sur les questions urbaines à une époque où ces problèmes sont particulièrement aigus, comme l'a bien montré T. Copp ${ }^{4}$, et où les forces sociales semblent s'affronter de manière privilégiée sur la scène montréalaise. Comment expliquer, notamment, la rareté des revendications en matière de logement? Comment comprendre le faible impact des victoires politiques ouvrières sur les politiques sociales locales? Comment, enfin, rendre compte de la précarité de ces victoires et de la débâcle politique complète qui s'ensuivit?

Ces questions mettent en jeu une double hypothèse de travail. D'une part, l'histoire du mouvement ouvrier ne peut être conduite indépendamment de celle des autres forces sociales. On verra plus particulièrement, dans le cadre de cet article, les interactions entre le mouvement ouvrier et les mouvements de réforme urbaine. D'autre part, nous voudrions montrer le rôle des facteurs proprement politiques dans la dynamique des rapports sociaux à Montréal. Car c'est bien autour de la scène politique montréalaise que se construisent, en interaction, le mouvement politique ouvrier et les mouvements de réforme urbaine (et plus particulièrement au sein de ceux-ci, le mouvement de réforme municipale).

Nous examinerons d'abord le contraste entre les victoires politiques ouvrières et la pauvreté du programme de revendications urbaines des organisations ouvrières. Nous verrons ensuite que ces revendications doivent être situées par rapport aux thèmes mis de l'avant par les mouvements de réforme urbaine, et que ces mouvements constituent une forme de réponse des classes supérieures à l'émergence d'une scène politique urbaine. L'analyse de ce contexte politique nous permettra alors de comprendre la précarité des victoires électorales ouvrières. Nous terminerons cet essai d'interprétation en nous interrogeant sur les enjeux sociaux des politiques sociales urbaines mises de l'avant par les réformistes avant les années ' $20^{5}$.

\footnotetext{
3 Le terme de «mouvement ouvrier» ne sera pas utilisé ici dans son sens sociologique fort de «mouvement social». Le concept de mouvement social a été défini par A. Touraine, notamment dans La voix et le regard (Paris, Seuil, 1978), deuxième partie.

4 T. Copp, Classe ouvrière et pauvreté - les conditions de vie des travailleurs montréalais, 1897-1929 (Montréal, Boréal Express, 1978).

5 Une bonne partie des analyses et des données présentées ici proviennent de l'historiographie sur les travailleurs québécois et de ma thèse de doctorat: A. Germain, Les mouvements de réforme urbaine à Montréal au tournant du siècle: modes de développement, modes d'urbanisation et transformations de la scène politique. Thèse de doctorat, Université de Montréal, département de Sociologie, 1980.
} 


\section{Les percées politiques des forces ouvrières à Montréal}

Les premiers chapitres de l'histoire du mouvement ouvrier organisé furent, jusqu'à un certain point, des chapitres «urbains». Sans retracer les grandes lignes de cette histoire aujourd'hui de mieux en mieux connue, rappelons brièvement les connotations urbaines du programme et de la clientèle des premières centrales ouvrières importantes au Québec.

Les Chevaliers idu Travail qui s'implantèrent au Québec dans les années 1880 avant d'être évincés par la montée d'un syndicalisme de métier (national et international), recrutaient le cinquième de leurs effectifs canadiens à Montréal. À la différence de leurs successeurs, les Chevaliers du Travail tentèrent de mobiliser toutes les couches de travailleurs: les ouvriers qualifiés et non qualifiés, les «sans travail» et les épouses des travailleurs ${ }^{6}$. Leur programme de revendications était aussi large que leur base sociale: loin de se cantonner au monde de l'usine, il englobait l'ensemble des conditions de vie des travailleurs. Convaincus de l'importance de la politique municipale dans l'aménagement de ces conditions de vie, les Chevaliers du Travail défendaient par ailleurs l'accessibilité des ouvriers au système politique. Ils revendiquèrent notamment une diminution de la qualification foncière exigée des candidats aux postes d'échevin et de maire, des heures de vote adaptées aux horaires des ouvriers, l'abolition de la corvée $\mathrm{e}^{7}$ dont le non-paiement faisait perdre le droit de vote, bref toutes mesures susceptibles de surmonter l'exclusion totale des masses populaires du système politique montréalais. La situation montréalaise contrastait à cet égard avec celle des villes américaines où dès le milieu du $\mathrm{XIX}^{\mathrm{e}}$ siècle, le suffrage était beaucoup plus largement répandu. Le principe de l'abolition de la corvée sera accepté dès 1883 au niveau municipal, mais les Chevaliers devront intenter un procès à la ville de Montréal et combattre le refus que leur impose la législature de Québec avant d'obtenir gain de cause ${ }^{8}$. Il faut toutefois se garder d'interpréter trop rapidement ces gains démocratiques comme autant de purs produits de conquêtes ouvrières. Car les pressions indéniables exercées par les Chevaliers se conjuguèrent avec les interventions d'élites politiques montantes et notamment de R. Préfontaine, maire de Montréal de 1898 à $1902^{9}$. Ces élites se serviront $\mathrm{du}$ potentiel politique que représentent les couches populaires canadiennes-françaises pour rétablir une majorité francophone à l'Hôtel de ville. En plus d'assouplir le système électoral, ces élites favoriseront

6 F. Harvey, «Les Chevaliers du Travail, les États-Unis et la Société québécoise. 18821902» in F. Harvey édit., Le mouvement ouvrier au Québec (Montréal, Boréal Express, 1980), 69-130.

J. de Bonville, Jean-Baptiste Gagnepetit: les travailleurs montréalais à la fin du XIX ${ }^{e}$ siècle (Montréal, L'aurore, 1975), 182-194.

8 Ibid., 186. 1969), 101. 
l'annexion à Montréal de municipalités ayant une majorité de résidents francophones (Hochelaga, Saint-Jean-Baptiste, Côte-Saint-Louis, Sainte-Cunégonde, Saint-Henri et Villeray). La géographie ethnique épouse suffisamment la division sociale de l'espace à Montréal pour que les questions de découpage territorial fassent l'objet d'enjeux politiues importants.

Lorsqu'à la toute fin du XIX $\mathrm{X}^{\mathrm{e}}$ siècle, les Chevaliers du Travail sont supplantés par des formes d'organisation syndicale plus modernes ${ }^{10}$, le mouvement ouvrier se durcit et s'institutionnalise. Sa présence politique se fait plus importante. Les grèves pour obtenir la reconnaissance syndicale se multiplient au début du siècle. La plupart de celles qui sont déclenchées à Montréal paralysent les secteurs les plus vitaux de la ville: le port, les services d'utilité publique (tramway, électricité), l'industrie lourde (usines Angus), le secteur de la construction et la fonction publique municipale. Par ailleurs, la plupart des quartiers de la ville voient éclore des clubs ouvriers municipaux. S'agit-il d'un héritage du mouvement des Chevaliers du travail, ou de groupes politiques bâtis sur le modèle des clubs politiques traditionnels, ou encore d'associations locales nées dans des quartiers ouvriers dès la fin du XIX siècle? L'origine sociale de ces clubs ouvriers n'est pas encore élucidée. $\mathrm{Au}$ début du $\mathrm{XX}^{\mathrm{e}}$ siècle ils émanent tantôt d'initiatives locales, à l'exemple des clubs politiques du parti libéral et du parti conservateur, tantôt des instances centrales du Parti ouvrier, tantôt des syndicats. Leur prolifération ainsi que la manière dont ces clubs s'identifient - nom d'une paroisse ou d'un quartier - , laissent supposer qu'une certaine «sédentarisation» des couches ouvrières s'est opérée, du moins en ce qui concerne les couches les plus qualifiées.

$\mathrm{Au} \mathrm{XIX}^{\mathrm{e}}$ siècle, une mobilité résidentielle élevée comme celle que constatait H.B. Ames dans sa City below the hill ${ }^{11}$ était à mettre au compte, d'une part, d'une structure industrielle dominée par l'industrie légère, c'est-à-dire par des emplois non qualifiés, précaires ou saisonniers ${ }^{12}$, et d'autre part, d'un sous-emploi produit par le décalage entre le rythme de croissance du marché du travail et le rythme de la

10 Selon J. Rouillard, les Chevaliers du Travail correspondaient à une forme de syndicalisme pré-industriel typique de petites entreprises familiales traditionnelles. Ils ne résisteront pas à la différenciation de la force de travail qu'engendre le capitalisme du $\mathrm{XX}^{\mathrm{e}}$ siècle et plus particulièrement à la montée des travailleurs qualifiés. De fait, les unions internationales et notamment le Congrès des métiers fédérés et du Travail de Montréal, affilié à la Fédération américaine du travail, ne recrutent que des ouvriers qualifiés afin de renforcer leur pouvoir de négociation face aux employeurs. J. Rouillard, Les syndicats nationaux au Québec de 1900 à 1930 (Québec, Presses de l'Université Laval, 1979), 7.

11 H.B. Ames, The City Below the Hill (Toronto, University of Toronto Press, 1972).

12 Les analyses de V. Shlakman sur l'industrie du vêtement sont intéressantes car cette industrie employait le quart de la main-d'oeuvre active montréalaise en 1881. V. Shlakman, Unemployment in the Men's Clothing Industries in Montreal, M.A. thesis, McGill University, 1931. Pour une analyse globale du problème du chômage saisonnier, voir L. Marsh: The Problem of Seasonal Unemployment, Ph.D. thesis, McGill University, 1933. 
croissance démographique urbaine. Contrastant avec la mobilité de ces couches ouvrières, la «sédentarisation» des ouvriers qualifiés, la fixation territoriale d'une population ouvrière sans doute plus urbanisée que les précédentes, aurait peut-être été propice au développement de nouvelles formes de sociabilité ouvrière illustrées par les clubs ouvriers municipaux ${ }^{13}$.

Enfin, il faut compléter la liste des victoires ouvrières en évoquant la mise sur pied du Parti ouvrier de Montréal en 1899, conformément aux résolutions adoptées par le Conseil des métiers et du travail du Canada et les clubs ouvriers de l'époque. On préfère à présent séparer nettement l'action syndicale et l'action politique, comme le veut la tradition américaine, afin d'éviter que «l'action politique partisane» ne soit source de division au sein des effectifs syndicaux. Se contentant, au début, d'accorder son appui à des candidats rattachés à d'autres formations politiques mais prêts à défendre son programme ${ }^{14}$, le Parti ouvrier ne commencera véritablement à fonctionner qu'en 1904. Et ce, avec une base de militants relativement diversifiée puisque les syndicats, les clubs ouvriers et la fraction socialiste dirigée par A. SaintMartin et G. Francq y sont affiliés.

En 1906, A. Verville, président du Conseil des métiers et du travail de Montréal est élu député de Maisonneuve au parlement fédéral. Dans son style bien particulier, R. Rumilly, témoin plus qu'historien (sic), commentait cet événement «historique» en ces termes:

C'est en somme la première élection «ouvrière» dans la province de Québec. Elle manifeste l'existence de cette grande force moderne: le syndicalisme. Les mécaniciens, les électriciens, les typographes, les cigariers, les plâtriers, les débardeurs et les employés du tramway, groupés dans leurs unions sous des chefs ouvriers, échappent aux grandes influences traditionnelles, peuvent dicter la loi dans la Ville de Montréal. ${ }^{15}$

Après 1906, la scène municipale devient la scène politique la plus importante pour le Parti ${ }^{16}$. En 1910 et en 1914, J. Ainey, président du Congrès des métiers fédérés et du travail, et candidat du Parti ouvrier, sera élu au poste de commissaire au Bureau de Contrôle de la Ville de Montréal (sorte de comité exécutif restreint, préparant et gérant le budget de la Ville). Il sera même candidat à la mairie en 1918 mais sera battu par M. Martin, futur grand «boss» de la politique montréalaise. J. Ainey sera également invité à siéger comme représentant ouvrier au

\footnotetext{
13 C'est du moins l'hypothèse que formulent certains historiens britanniques à propos de Londres. G. St-Jones, Outcast London (London, Oxford University Press, 1971).

14 En fait de programme, les organisations ouvrières insisteront surtout sur le principe de l'abolition de la qualification foncière pour les postes d'échevin et de maire.

15 R. Rumilly, Histoire de Montréal (Montréal, Fides, 1972), tome 3: 369.

16 J. Rouillard, «L'action politique ouvrière au début du $20^{\mathrm{e}}$ siècle» in F. Harvey, édit.: Le mouvement ouvrier au Québec, op. cit.
} 
Montreal Board of Trade et Verville à la Commission des Tramways. C'est dire le poids politique que représentent les forces ouvrières à Montréal.

Ces victoires politiques ne se traduiront pas nécessairement en termes de programmes d'amélioration des conditions de vie. D'ailleurs, parmi les revendications ouvrières en matière urbaine, celles qui ne sont pas intrinsèquement politiques, sont fort peu nombreuses.

Si l'on regarde les thèmes sur lesquels font campagne les candidats soutenus par le Parti ouvrier, la Fédération des clubs ouvriers municipaux (regroupant les clubs qui ont soutenu des candidats non endossés par le Parti) et le Congrès des métiers et du travail de Montréal, et plus largement les revendications formulées par l'ensemble des organisations ouvrières sur la scène politique ${ }^{17}$, un certain nombre de constatations s'imposent.

Jusqu'à la fin de la deuxième décennie, des thèmes comme l'augmentation du coût de la vie, le chômage, la création de dispensaires de soins médicaux gratuits, l'organisation de potagers urbains, mobiliseront les organisations ouvrières de façon épisodique. Les revendications concernant le logement et l'hygiène sont beaucoup plus rares, surtout avant la guerre. Sur le plan du logement, les syndicats et les clubs ouvriers se joignent au mouvement dirigé par diverses organisations (comme les Chambres de commerce et la Ligue du Progrès civique) pour abolir le système de location annuelle obligatoire afin d'y substituer la formule, plus souple, des baux mensuels. Un des principaux protagonistes de cette campagne, le docteur Adami, président de la Ligue du Progrès civique, expliquera que le système des baux annuels désavantage les ouvriers parce qu'il entrave leur mobilité au niveau du marché de l'emploi ${ }^{18}$. En outre le programme du Parti ouvrier de 1899 comprenait un article sur la création d'une caisse de prêt pour aider les ouvriers à s'acheter une maison. Cet article ne sera cependant pas repris dans les programmes ultérieurs ${ }^{19}$. Tout se passe donc comme si la question du logement n'était pas encore une «question sociale», du moins pour les catégories ouvrières représentées par le mouvement ouvrier.

Par contre, les problèmes relatifs aux services d'utilité publique semblent mobiliser davantage les organisations ouvrières. Entre 1906

\footnotetext{
17 Regroupement des chercheurs en histoire des travailleurs québécois: Chronologie des mouvements politiques ouvriers au Québec de la fin du XIX siècle à 1919 (Montréal, 1976), non publié.

8 La Presse, 10 mars 1910. La ligue du progrès civique et le Montreal Board of Trade utiliseront le même argument de la mobilité, dans les années ' 30 , pour se prononcer contre les politiques d'accession à la propriété du logement.

Cet article réapparaîtra dans les programmes des années '20, donc après la guerre, les autres problèmes liés au logement semblant disparaître derrière cette unique préoccupation de l'accession à la propriété du logement. Fr. Charbonneau, La question du logement à la C.S.N. (Montréal, C.S.N.), dossier logement no 4, document de travail non publié, mai 1981.
} 
et 1910, les unions internationales et le Parti ouvrier se prononceront en faveur de la municipalisation des tramways et des services de distribution de gaz et d'électricité. En 1915, toutefois, le Conseil des métiers et du travail de Montréal se rétractera et réclamera l'extension du réseau des tramways plutôt que sa municipalisation. Plusieurs clubs ouvriers municipaux interviendront également dans ce dossier, pour s'opposer notamment aux velléités du gouvernement provincial de se substituer aux autorités municipales, dans le but de «régler la question des tramways». En 1911, J. Ainey participera à une assemblée publique organisée par le maire réformiste J.-J. Guérin, pour protester contre le contrat exclusif d'exploitation sollicité par la Compagnie des tramways auprès de la législature.

De fait, dans les dossiers relatifs aux services d'utilité publique, les organisations ouvrières emboîtent le pas aux «mouvements de réforme urbaine», plus qu'elle ne prennent l'initiative de campagnes de revendications originales. Il est donc important de situer les luttes politiques ouvrières par rapport à ces mouvements de réforme. On verra d'ailleurs ultérieurement le rôle joué par ces derniers dans la débâcle des forces politiques représentant les ouvriers.

\section{Les mouvements de réforme urbaine}

En effet, il faut nettement distinguer ces mouvements des organisations ouvrières. Au début, ils sont le fait de membres des classes dirigeantes anglophones mais leur base sociale se diversifiera par la suite, sans pour autant inclure de nombreux représentants des classes populaires. Comme dans la plupart des grandes villes nord-américaines, ces mouvements sont composés de «comités de citoyens» qui se forment à partir des années 1880 autour de thèmes aussi variés que la réforme de l'administration municipale, l'urbanisme, l'hygiène, le contrôle des monopoles dans les services d'utilité publique, la participation des femmes à la vie publique, les terrains de jeux pour les enfants des quartiers pauvres, etc. ${ }^{20}$ De nombreux dossiers déboucheront sur des projets qui resteront lettre morte. C'est le cas, entre autres, de l'urbanisme: le plan de Montréal proposé par l'Association des architectes en $1909^{21}$, les projets de tramways souterrains (métro) ${ }^{22}$, de cités-jardins, de boulevards et de monuments publics ${ }^{23}$, ne dépasseront guère

20 Parmi les comités les plus actifs citons La ligue du Progrès civique de Montréal, the Pure Milk League, the Montreal Parks and Playground Association, the Montreal Local Council of Women, the Montreal Anti-Tuberculosis League, la Fédération nationale St-Jean-Baptiste, etc. Pour une description des mouvements de réforme urbaine à Montréal et, plus largement, en Amérique du Nord, je me permets de renvoyer le lecteur aux trois premiers chapitres de ma thèse de doctorat.

${ }_{21}$ Premier Rapport annuel de la Ligue du Progrès civique de Montréal (Montréal, 1910).

22 Montreal Herald, march 10, 1910; La Presse, 1, 2, 4 mars 1910 et 14, 22, 26 avril 1910, 16 janvier 1914, 16 mai 1914. 21,1910 .

La Presse, 21, 26, 29, 30 mars 1910, 16 et 26 août 1910; Montreal Herald, march 12, 
le stade des esquisses vagues mais combien spectaculaires. Par contre, en banlieue, des villes comme Maisonneuve, Westmount, Hampstead et Ville Mont-Royal réaliseront des projets d'aménagement faisant écho aux nouveaux courants d'urbanisme. En matière de politique sociale montréalaise, les réalisations sont l'exception plutôt que la règle. On cite souvent les campagnes menées par l'industriel H.B. Ames pour améliorer les conditions de logement des ouvriers. Mais les logements construits par Ames, à Diamond Court ${ }^{24}$, dans une politique de «philanthropie plus $5 \%$ de profit» ne sont pas du tout représentatifs des pratiques de la bourgeoisie montréalaise. La bourgeoisie montréalaise a certes un réseau important d'associations philanthropiques, comme il est de bon ton à l'époque, mais il y a des secteurs dans lesquels elle n'intervient pratiquement pas. Le logement ouvrier en est un. On pourrait d'ailleurs faire l'hypothèse que l'absence d'une bourgeoisie industrielle forte au sein des classes supérieures montréalaises expliquerait, en partie, le peu d'initiatives prises par celles-ci dans l'amélioration et le contrôle des conditions de vie des classes laborieuses ${ }^{25}$. L'histoire sociale comparée nous a appris que les réformes sociales sont rarement promues par une bourgeoisie mercantile et financière comme celle qui dominait Montréal au XIX ${ }^{\mathrm{e}}$ siècle $^{26}$. On sait aussi que la présence ou l'absence de classes moyennes prêtes à lier leur avenir à un élargissement des champs d'intervention de l'État constitue un autre facteur décisif dans la genèse de politiques sociales. À Montréal, la pérennité des systèmes d'encadrement traditionnels (Église et organisations charitables diverses) a certainement pesé sur le peu d'ouverture des mouvements de réforme montréalais aux impératifs de la prise en charge des problèmes sociaux urbains.

Le principal cheval de bataille des réformistes sera, avant tout, la réforme de l'administration municipale. Menant de rudes batailles contre les «bosses» et leur machine politique pour dénoncer la corruption de l'administration municipale, les associations réformistes prôneront des principes d'économie et de rationalité dans la gestion des affaires urbaines ainsi que la dépolitisation de l'administration municipale. Les

24 J.M. Wolfe, The Progress of Housing Reform; three early experiments in Montreal (Montreal, School of Urban Planning, McGill University, Working paper, february 1981), 2-6.

25 Plusieurs recherches en cours tentent de cerner le fonctionnement du secteur de la construction résidentielle. Les difficultés de trouver du financement à bon marché n'auraient peut-être pas empêché la prolifération de petits constructeurs et de promoteurs urbains de différents acabits. Par ailleurs le constat des conditions de vie extrêmement pénibles sévissant dans certains quartiers ouvriers de la ville, n'autorise cependant pas l'établissement de rapprochements entre une ville comme Londres et Montréal, cette dernière n'ayant pas connu une pénurie de logement aussi importante que la précédente.

${ }_{26}$ Sur ce plan, certaines analogies peuvent être faites avec la structure sociale londonienne. Par exemple, J. Foster, parlant de la bourgeoisie financière londonienne, dira: «... they were not concerned with how to ensure the extended reproduction of labour power... their main concern was how to prevent the social costs of such reproduction halting the entire process by which their own rentier incomes were reproduced». J. Foster, «How Imperial London Preserved Its Slums», Journal of International Urban and Regional Research, 3, 1 (march 1979): 103. 
conflits politiques étaient assimilés à des luttes dites «partisanes» entravant le déroulement des processus administratifs. Car la gestion urbaine ne devait pas être une «affaire politique». Dans l'esprit des réformistes, le gouvernement municipal n'était donc pas un gouvernement comme ceux de la province et de la nation.

The making of by-law and the other strictly legislative function of a city council have, nowadays, become things of secondary importance. The modern city is first and foremost a guardian of public order, public health and private property, a builder of streets, parks, sewers and the other agencies of public convenience, a purveyer of water and light, a regulator of transportation. All these things involve problems of business and not of government. ${ }^{27}$

La ville devait être gérée comme une entreprise, en séparant nettement l'exécutif, le législatif et l'administratif. Ainsi le «bureau de contrôle» proposé par les réformistes fonctionnerait de manière distincte du conseil municipal, à l'instar d'un conseil d'administration.

Auparavant, les associations de réforme municipale, comme celle mise sur pied par le Board of Trade dès 1886, avaient tenté de freiner l'influence grandissante des échevins représentant les quartiers de l'est de la ville. Après avoir tenté, en vain, d'éliminer l'importance des quartiers en tant que districts électoraux, ils obtiendront à plusieurs reprises le gel de la dette de la ville, afin d'arrêter les programmes de travaux publics destinés à ces quartiers de l'est de Montréal ${ }^{28}$.

En prenant du recul, on s'aperçoit que la fonction sociale de ces mouvements de réforme était avant tout de nature politique. L'urbanisation et l'industrialisation avaient profondément transformé la ville. Montréal n'était plus une société victorienne où pouvoir politique et pouvoir économique se confondaient. Si les réformistes plaidaient le retour à un gouvernement des hommes d'affaires, c'est que précisément Montréal était devenue une scène politique authentique.

\section{3. Émergence d'une scène politique}

La notion de scène politique désigne l'espace social où se rencontrent, sur le mode du conflit ou de la négociation, les forces politiques mises en présence dans la prise de décisions légitimes. Il n'existe pas de scène politique lorsque les décisions sont prises et imposées pà les classes dominantes, sans qu'aucune autre force sociale ne soit représentée ou participe au processus. Tel était le cas de Montréal au XIX

27 W.B. Munro, «City Government in Canada: Boards of Control and Commission Government in Canadian Cities», Canadian Political Science Association, Proceedings and Papers, September 5, 1913, 112 (les soulignés sont de l'auteur).

28 M. Gauvin, The Municipal Reform Movement in Montreal, 1896-1914, M.A. thesis, University of Ottawa, 1972. 
siècle lorsqu'une grande bourgeoisie mercantile y confondait le contrôle du système urbain et la conduite de ses affaires commerciales.

En quelques décennies, on assistera à l'ouverture du système politique dans la mesure où les masses n'en seront plus totalement exclues. Après la guerre de 1914, l'incorporation politique tardive de ces masses fera, en partie, le succès de ces maires populistes que seront les «bosses» Médéric Martin et Camillien Houde. La participation aux élections municipales augmentera de façon spectaculaire à partir de 1914. On notera également de profonds changements dans l'évolution de l'origine sociale du personnel politique montréalais, la politique municipale n'étant plus l'apanage de la bourgeoisie ${ }^{30}$. Le «bossisme» doit alors être analysé autant comme un style de gouvernement manipulateur et un mécanisme d'intégration des masses populaires que comme l'expression des pressions de ces masses sur la scène politique.

En plus de se caractériser par son ouverture, le système politique montréalais du début du siècle devient aussi plus autonome par rapport aux grands clivages sociaux. En d'autres termes, les divisions entre les forces politiques ne reflètent pas nécessairement des conflits entre classes sociales, les intérêts politiques ne traduisant pas nécessairement des intérêts socio-économiques. La scène municipale fait figure de marché politique sur lequel les alliances et les clivages politiques semblent fluides, variant au gré des conjonctures. Par exemple, les grandes entreprises de services d'utilité publique se rangeront alternativement sous la bannière réformiste (les réformistes ayant pourtant critiqué leurs tendances monopolistiques) et sous celle du «boss» de l'époque. Comme l'ouverture du système politique est contemporaine de l'éclatement progressif du mouvement ouvrier, le jeu politique semble précipiter la désunion des forces ouvrières. On verra par exemple les syndicats nationaux soutenir la candidature du «boss» M. Martin contre celle du candidat ouvrier désigné par les unions internationales. Ces derniers soutiendront $\mathrm{M}$. Martin, à leur tour, quelques années plus tard, après avoir bénéficié de son appui. Chaque élection donne lieu à des tensions et à des scissions au sein du mouvement ouvrier. La Fédération des clubs ouvriers municipaux se forme comme organisation dissidente du Parti ouvrier, certains clubs ouvriers ayant soutenu des candidats non endossés par le Parti. Elle finira par supplanter le Parti en 1912 avant de disparaître elle-même du paysage politique après la guerre au profit de la Fédération des clubs ouvriers de surveillance politique.

Si la scène municipale locale est susceptible de servir de tremplin à des forces politiques représentant des groupes sociaux non organisés, elle pose aux forces sociales organisées de sérieux dilemmes. Car la

\footnotetext{
30 G. Bourassa, «Les élites politiques de Montréal: de l'aristocratie à la démocratie», Canadian Journal of Economic and Political Science. Revue canadienne de sciences économiques, sociales et politiques, XXXI (february-février 1965): 35-51.
} 
participation au système politique et la représentation des forces sociales sur la scène politique sont souvent traversées par des exigences opposées $^{31}$. Les petites classes moyennes traditionnelles (petits entrepreneurs, petits commerçants etc.) sont très présentes dans le système politique montréalais du début du $\mathrm{XX}^{\mathrm{e}}$ siècle: elles y participent par le biais du patronage, en obtenant des contrats et des privilèges de l'administration municipale. Mais elles ne sont pas représentées comme telles à l'Hôtel de Ville. Par contre d'autres groupes sociaux obtiennent d'être représentés sur la scène politique mais ne réussiront pas à infléchir l'orientation des politiques municipales.

La cooptation des chefs ouvriers par les réformistes au Bureau de contrôle de la ville de Montréal constitue un bel exemple de ce genre de dilemme. Selon le sociologue Ph. Selznick, la cooptation est un mécanisme qui consiste à absorber de nouveaux éléments dans la structure de direction d'une organisation dans le but d'éviter les menaces que représentent ces éléments pour sa stabilité ou son existence ${ }^{32}$. Telle est bien la manière dont les réformistes vont neutraliser les représentants politiques ouvriers.

Voyons cela plus en détail. Nous savons qu'en 1909 les associations réformistes réussissent à imposer (à la suite d'un référendum) l'idée d'instaurer un bureau de contrôle à Montréal. En 1910, l'Association des citoyens présente comme candidats aux quatre postes de commissaires des personnalités représentant divers milieux: J. Ainey, candidat du Parti ouvrier; L. Dupuis, commerçant réputé et grand propriétaire foncier; F. Wanklyn, ingénieur civil et ancien gérant du GrandTronc, puis de la Montreal Street Railway Co., vice-président de la Dominion Coal Co.; et E.-P. Lachapelle, médecin, directeur du Bureau provincial d'hygiène, président du Crédit foncier et de la Banque provinciale et sympathisant réformiste ${ }^{33}$. Le candidat réformiste à la mairie est J.-J. Guérin, un médecin irlandais. Tous ces candidats seront élus. Plusieurs chefs ouvriers avaient auparavant exprimé leurs craintes visà-vis de cette nouvelle institution. Le socialiste G. Francq n'avait-il pas déclaré que le projet de Bureau de contrôle n'était qu'un complot ourdi par de grands capitalistes ${ }^{34}$. De fait, la participation de J. Ainey au bureau de contrôle permettra d'asseoir la légitimité de ce nouvel organisme. Elle constituera aussi un excellent moyen d'entraver l'expression politique des intérêts de la classe ouvrière. Elle obligera notamment Ainey à endosser un principe de neutralité excluant précisément la représentation politique d'intérêts sociaux particuliers. Ainsi déclarera-t-il:

\footnotetext{
31 A. Touraine, Production de la société (Paris, Seuil, 1973), 220-223.

32 Ph. Selznick, T.V.A. and the Grass Roots Democracy (N.Y., Harper Torchbooks, 1966).

33 R. Rumilly, op. cit.: 441.

34 M. Gauvin, op. cit.
} 
... but what I object to most of all in all these discussions is the bringing in of the working class. What have the working class or any other class got to do with the way we administer the affairs of the city? We are not administering the city of any particular class. ${ }^{35}$

En adoptant ce langage de l'intérêt public, Ainey reprend à son compte les idées que nourrissent les réformistes par rapport à la politique locale.

Le programme avec lequel $\mathbf{J}$. Ainey se présentera à l'élection municipale de 1918, reprendra de nombreux points du programme réformiste. En effet, sur les dix (10) articles composant le programme, sept (7) s'inspirent des revendications réformistes ${ }^{36}$. Lors de son premier mandat, le CMTM l'avait blâmé pour avoir accepté un projet de réduction des salaires des employés municipaux, projet qui suscitera d'ailleurs la grève des policiers, des pompiers et des éboueurs. En 1916, la Fédération des clubs ouvriers municipaux réclamera l'abolition du Bureau de contrôle, alors que cette formule politico-administrative vient, précisément, de recevoir l'assentiment du CMTM. En somme, la participation d'Ainey au Bureau de contrôle précipitera la débâcle et la division des forces ouvrières plus qu'elle ne les retardera.

À tout prendre, les enjeux urbains qui n'ont pas une connotation directement politique semblent toutefois aussi secondaires pour les organisations politiques ouvrières que pour les associations réformistes. Même le maire J.-J. Guérin, représentant notoire du mouvement hygiéniste, ne réussira pas à concrétiser ses idées en matière de santé publique. Serait-ce donc, pour reprendre un thème cher à S.B. Warner, que l'institution municipale fonctionne davantage comme une institution représentative que comme une institution de gestion des affaires urbaines?

During the nineteenth century the civic community devoted itself predominantly to real estate services: streets, water, sewers, police and fire protection, and the cultural activity of education. The second function of the civic community is representation; the representation of the classes and the varying cultures... in America, the representative task of the civic community generates more popular concern and enthusiasm than the services, and the largest voter

35 Cité par M. Gauvin, op. cit., 89.

36 Les articles du programme électoral de J. Ainey, qui reprennent les revendications réformistes sont les articles $1,2,3,6,7,8$ et 9-1. Harmonie et bonne entente entre le Conseil et le Bureau des administrateurs. 2. Administration des affaires municipales sur des bases d'affaires. 3. Plan d'ensemble pour le Montréal d'aujourd'hui et le Grand Montréal de demain. 4. Extension de la bibliothèque publique par la création de succursales dans toutes les parties de la ville. 5 . Développement du système des marchés, dans le but de réduire le coût de la vie. 6 . Règlement de la question des abattoirs. 7. Développement de nos parcs et terrains de jeux. 8. Création d'une commission de service civil. 9. Améliorer le service de l'eau et l'alimentation au meilleur marché possible pour toute la ville. 10. Salaire pour les hommes de métier suivant le tarif des unions ouvrières. Reproduit dans L'action politique des ouvriers québécois, fin du $19^{e}$ siècle, Recueil de Documents (Montréal, Presses de l'Université du Québec, 1976), 102. 
turn-outs and greatest political conflict have taken place in the realm of representation. ${ }^{37}$

$\mathrm{Au} \mathrm{XIX}^{\mathrm{e}}$ siècle, les questions relatives aux dossiers stratégiques en matière de développement urbain étaient débattues, au niveau local, à l'extérieur des institutions municipales, au sein d'organisations telles que le Montreal Board of Trade, la Commission du Havre ou les nombreux clubs privés au sein desquels se rencontraient les membres de la grande bourgeoisie montréalaise. Lorsqu'au tournant du siècle, le système politique montréalais s'ouvre, sous la pression conjuguée des forces ouvrières et des nouvelles élites canadiennes-françaises, ce sont des enjeux politiques qui cristallisent les affrontements entre différentes forces politiques. La scène politique montréalaise semble servir de tremplin aux organisations ouvrières, leur permettant d'affirmer la présence de cette nouvelle force sociale que sont les ouvriers. Mais l'action politique n'aura pas pour mission de débattre des orientations du développement urbain.

Par ailleurs, plus l'institution municipale sera affaiblie, plus on retirera de l'arène municipale des dossiers chauds (en plaçant par exemple sous les auspices du gouvernement provincial la négociation des contrats d'exploitation des services d'utilité publique ${ }^{38}$, moins elle sera une plate-forme privilégiée de débats urbains. Les réformistes (parmi lesquels on compte les principaux représentants des milieux financiers montréalais) n'auront de cesse de réclamer la limitation des dépenses du gouvernement municipal, afin d'empêcher ce dernier d'exercer des fonctions de redistribution sociale ${ }^{39}$. Même H.B. Ames, qui se sera tant battu pour améliorer le sort de ses ouvriers, se montrera toujours opposé à toute intervention de l'État en la matière, au-delà de strictes mesures de réglementation: «I am not an advocate for experiments in housing and lodging on the part of our civic authorities. They have no right to take chances with the people's money.» ${ }^{40}$

\footnotetext{
37 S.B. Warner, Streetcar Suburbs: The Process of Growth in Boston 1870-1900 (Cam-

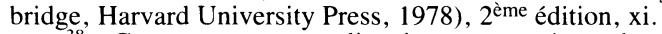

38 Cette mesure «centralisatrice» que représente la création de la Commission des tramways par le gouvernement provincial n'est pas isolée: la création du ministère des Affaires municipales en 1918 dans le but de contrôler les finances des municipalités, la mise en tutelle de la ville de Montréal la même année, ainsi que les premières institutions métropolitaines telles que la Commission métropolitaine des parcs de Montréal, puis la Commission métropolitaine de l'île de Montréal (ayant pour tâche de contrôler les emprunts des municipalités) représentent autant de manières de rétrécir le champ des décisions municipales et donc d'affaiblir l'institution municipale.

Lorsqu'en 1918, le gouvernement fédéral adoptera une loi sur l'habitation au titre des mesures de guerre afin de stimuler la construction d'habitations, et que le Québec votera la «Loi pourvoyant à la construction de logements ouvriers et à des avances aux municipalités», la Commission administrative, alors en charge des affaires de Montréal, refusera de se prévaloir de cette loi. Ce refus doit être situé dans l'esprit de la politique d'austérité pratiquée par la Commission, afin de redresser la situation financière de la ville.

40 H.B. Ames, "The housing of the Working Class», Canadian municipal Journal, VI, 10 (oct. 1977): 25 .
} 
Enfin, lorsque, dans le sillage du mouvement urbaniste qui se développe dans les années ' 10 , le mouvement réformiste se penchera sur des projets de logement, ce ne sera plus véritablement de logement ouvrier qu'il s'agira. Par exemple, les projets de «suburban community planning» que P. Nobbs expose aux membres de la Ligue du Progrès civique ont pour cadre rien de moins que la ville de Hampstead, l'une des banlieues les plus riches de la région montréalaise ${ }^{41}$. Et lorsqu'en 1911 le maire pro-réformiste J.-J. Guérin évoque les projets de citésjardins que prépare son administration, il prend soin de préciser quels seront les bénéficiaires de ces projets:

It must be understood that these new Garden city Dwellings are not intended to replace the slums and disease infected districts which the new east and west boulevards will wipe out... The idea of the Garden City is more to provide a comfortable and pretty home for the mechanic and the clerk than to make things easy for those who choose to disregard all the natural and physical laws in existence, maintaining a hand to mouth existence by day, and retiring to their dark, noisesome, rooms at night huddled together, six, eight and ten in a room. ${ }^{42}$

De fait, toutes les politiques et tous les projets privés de logement ouvrier seront toujours porteurs d'ambiguïté quant aux catégories sociales bénéficiaires: classes ouvrières ou classes moyennes? ${ }^{43}$

\section{Conclusion}

Bien que la scène politique montréalaise soit, au tournant du siècle, une plate-forme privilégiée où s'affrontent les forces sociales et vers laquelle sont refoulés les problèmes sociaux majeurs de l'époque, elle n'en est pas pour autant le cadre des débats sociaux qui auraient la gestion de ces problèmes pour objet. Les forces ouvrières qui réussissent à se faire représenter sur la scène montréalaise seront rapidement entraînées dans des processus politiques qui entravent l'expression des intérêts de la classe ouvrière plus qu'ils ne la favorisent. C'est là un effet du système politique plus que d'une manipulation délibérée par les forces dominantes. Dans la lutte opposant organisations ouvrières et mouvements réformistes, le passage d'une logique de la représentation à une logique de la participation au système politique s'avéra fatal pour le mouvement ouvrier. D'ailleurs, l'idée d'une force ouvrière organisée pose le problème de sa représentativité par rapport à l'ensemble des couches populaires. Nous avons vu les organisations politiques et syndicales se diviser et faire des choix divergents en matière de politique municipale. Mais ces organisations recrutaient leurs membres

\footnotetext{
41 P.E. Nobbs: Suburban Community Planning, McGill University Publications Series, XIII, 7 (1926)

42 The Montreal Herald, december 23, 1911.

43 Sur ce point, voir A. Germain, op. cit., chapitre VII.
} 
parmi les travailleurs qualifiés plus souvent qu'autrement. Les succès de cette forme de populisme ${ }^{44}$ que représente le «bossisme» à Montréal laisse penser que des maires comme M. Martin et C. Houde ralliaient des couches populaires qui restaient largement en marge du mouvement politique organisé. Quelles différences existaient-ils dans les conditions et modes de vie des différentes catégories d'ouvriers? Les problèmes de logement se posaient-ils de la même manière pour tous les travailleurs? Les réponses à de telles questions me semblent conditionner l'évaluation que l'on peut faire de l'action politique ouvrière à Montréal.

44 Le populisme est une forme d'exercice du pouvoir politique qui fonctionne sur base d'une idéologie opposant les "petits» (le peuple) à un adversaire démesuré et généralement étranger à la communauté (par exemple: les millionnaires anglophones). Il est caractéristique de situations de «masse», c'est-à-dire de situations dans lesquelles des groupes sociaux n'ayant pas d'emprise sur leur environnement ne peuvent s'y constituer en acteurs sociaux autonomes. 